\title{
CONTINUITY OF PLURISUBHARMONIC ENVELOPES IN $\mathbb{C}^{2}$
}

\author{
NİHAT GÖKHAN GÖĞÜŞ AND SÖNMEZ ŞAHUTOĞLU
}

\begin{abstract}
We show that in $\mathbb{C}^{2}$ if the set of strongly regular points are closed in the boundary of a smooth bounded pseudoconvex domain, then the domain is $c$-regular, that is, the plurisubharmonic upper envelopes of functions continuous up to the boundary are continuous on the closure of the domain. Using this result we prove that smooth bounded pseudoconvex Reinhardt domains in $\mathbb{C}^{2}$ are $c$-regular.
\end{abstract}

\section{INTRODUCTION}

Let $\Omega$ be a domain in $\mathbb{C}^{n}$ and $\partial \Omega$ denote the boundary of $\Omega$. For any upper bounded function $u$ on $\Omega$ we define the upper regularization $u^{*}$ of $u$ on $\bar{\Omega}$ as the function

$$
u^{*}(z)=\limsup _{w \in \Omega, w \rightarrow z} u(w)
$$

for any $z \in \bar{\Omega}$. Given $z \in \bar{\Omega}$, we denote by $\mathcal{J}_{z}=\mathcal{J}_{z}(\Omega)$ the family of all positive Borel measures $\mu \in C^{*}(\bar{\Omega})$ such that $u^{*}(z) \leq \int u^{*} d \mu$ for every $u$ in the set $P S H^{b}(\Omega)$ of all upper bounded plurisubharmonic functions on $\Omega$. Such measures are called Jensen measures centered at $z$. For example, the Dirac measure $\delta_{z} \in \mathcal{J}_{z}$ for every $z \in \bar{\Omega}$. For a nontrivial example, let $z \in \Omega$ and consider a map $f: \overline{\mathbb{D}} \rightarrow \Omega$ which is holomorphic in an open neighborhood of the closure of the unit disk $\overline{\mathbb{D}}$ so that $f(0)=z$. Define

$$
\mu_{f}(\varphi)=\frac{1}{2 \pi} \int_{0}^{2 \pi} \varphi \circ f\left(e^{i \theta}\right) d \theta
$$

for any function $\varphi \in C(\bar{\Omega})$. Then $\mu_{f}$ defines a measure on $\Omega$ which belongs to $\mathcal{J}_{z}$. Let us denote the class of all measures of the form $\mu_{f}$, where $f$ is a mapping as above, by $\mathcal{H}_{z}$. It was proved in [2] that the weak-* closure $\overline{\mathcal{H}}_{z}$ in $C^{*}(\bar{\Omega})$ of $\mathcal{H}_{z}$ coincides with $\mathcal{J}_{z}$ when $z \in \Omega$. Moreover, by [12] and [13] if $\varphi$ is an upper semicontinuous function on $\Omega$, then

Date: November 6, 2018.

2010 Mathematics Subject Classification. Primary 32U15.

Key words and phrases. Jensen measure, plurisubharmonic envelopes, Reinhardt domains.

The first author is supported by the Scientific and Technological Research Council of Turkey related to a grant project called "Jensen measures in complex analysis and c-regularity" with project number 110T223. 
the function

$$
I \varphi(z)=\inf \left\{\int \varphi d \mu: \mu \in \mathcal{H}_{z}\right\}
$$

is plurisubharmonic and equal to the plurisubharmonic upper envelope $E \varphi$ of $\varphi$ on $\Omega$, where

$$
E \varphi(z)=\sup \{u(z): u \leq \varphi \text { and } u \text { is plurisubharmonic on } \Omega\} .
$$

We define the class of measures $\widehat{\mathcal{J}}_{z}$ for $z \in \bar{\Omega}$ in the following way: A measure $\mu \in$ $C^{*}(\bar{\Omega})$ belongs to $\widehat{\mathcal{J}}_{z}$ if there exist points $z_{j} \in \Omega$ and measures $\mu_{j} \in \mathcal{J}_{z_{j}}$ such that $\left\{z_{j}\right\}$ converges to $z$ and $\left\{\mu_{j}\right\}$ converges to $\mu$ in the weak-* topology. A point $z \in \partial \Omega$ is said to be c-regular if the following holds: If $z_{j} \in \Omega, z_{j} \rightarrow z$ and $\mu \in \widehat{\mathcal{J}}_{z}$, then there exists a sequence of measures $\mu_{j} \in \mathcal{J}_{z_{j}}$ that converges weak-* to $\mu$. A domain is called $c$-regular if every boundary point is $c$-regular. The following theorem establishes the fact that regularity of plurisubharmonic envelopes is equivalent to $c$-regularity of the domain (see [7, 8] $)$.

Theorem 1 (Göğüss). Let $\Omega$ be a bounded domain in $\mathbb{C}^{n}$. All points in the boundary of $\Omega$ are c-regular if and only if the envelope $E \varphi$ is continuous on $\bar{\Omega}$ for all functions $\varphi \in C(\bar{\Omega})$.

Plurisubharmonic upper envelopes of functions form an important tool in pluripotential theory. One uses them in particular to construct homogeneous solutions of the complex Monge-Ampère operator with continuous boundary data. In [7] the first author used Jensen measures to completely characterize the domains where continuous functions have continuous envelopes. For any function $\psi \in C(\partial \Omega)$, the Perron-Bremermann function for $\psi$ is the function $S \psi$ defined on $\bar{\Omega}$ by

$$
S \psi(z)=\sup \left\{u^{*}(z):\left.u^{*}\right|_{\partial \Omega} \leq \psi \text { and } u \text { is plurisubharmonic on } \Omega\right\} .
$$

It is a well-known result due to Bremermann [1], Walsh [16], Bedford and Taylor [3] that when $\Omega$ is a bounded strongly pseudoconvex domain in $\mathbb{C}^{n}$, given a function $\psi \in C(\partial \Omega)$, the function $S \psi$ is maximal plurisubharmonic in the sense that $\left(d d^{c} S \psi\right)^{n}=0$ on $\Omega, S \psi$ is continuous, and also $\lim _{w \rightarrow z} S \psi(w)=\psi(z)$ for every $z \in \partial \Omega$. Thus $S \psi$ is the unique homogeneous solution of the complex Monge-Ampère operator with continuous boundary data $\psi$ when the domain is strongly pseudoconvex. Related to this, it was proved in [7] that c-regularity of the domain is equivalent to the continuity of Perron-Bremermann envelopes on smooth bounded pseudoconvex domains in $\mathbb{C}^{n}$.

Theorem 2 (Göğüss). Let $\Omega$ be a smooth bounded pseudoconvex domain in $\mathbb{C}^{n}$. Then all points in the boundary of $\Omega$ are c-regular if and only if the Perron-Bremermann function $S \psi$ is continuous on $\bar{\Omega}$ for every $\psi \in C(\partial \Omega)$. 
A point $p \in \partial \Omega$ is said to be strongly regular if there exists a function $u \in \operatorname{PSH}(\Omega) \cap$ $C(\bar{\Omega})$ so that $u(p)=0$ and $u(z)<0$ for every $z \in \bar{\Omega} \backslash\{p\}$. The motivation for this paper comes from the following theorem of the first author [7].

Theorem 3 (Gögüuss). Let $\Omega$ be a bounded domain in $\mathbb{C}^{n}$. Assume that the boundary of $\Omega$ is c-regular. Then the set of strongly regular points is a closed subset of the boundary of $\Omega$.

In this paper we prove that Theorem 3 has a converse on smooth bounded pseudoconvex domains in $\mathbb{C}^{2}$ and that smooth bounded pseudoconvex Reinhardt domains in $\mathbb{C}^{2}$ are $c$-regular (see Theorem 4 and Theorem 5 in the next section).

The rest of the paper is organized as follows. The main results are given in the next section. After that we give two examples to show that one cannot hope to have similar results as in Theorem 4 and Theorem 5 in $\mathbb{C}^{n}$ for $n \geq 3$. We postpone the proofs of the results until after the examples. We conclude the paper with some remarks.

\section{RESULTS}

Our first result states that Theorem 3 has a converse on smooth bounded pseudoconvex domains in $\mathbb{C}^{2}$.

Theorem 4. Let $\Omega$ be a smooth bounded pseudoconvex domain in $\mathbb{C}^{2}$. Then $\Omega$ is c-regular if and only if the set of strongly regular points is closed in the boundary of $\Omega$.

Combining Theorem 11, Theorem 2 and Theorem 4 we get the following corollary.

Corollary 1. Let $\Omega$ be a smooth bounded pseudoconvex domain in $\mathbb{C}^{2}$. Then the following statements are equivalent:

i) The set of strongly regular points is closed in the boundary of $\Omega$.

ii) The envelope $E \varphi$ is continuous on $\bar{\Omega}$ for all functions $\varphi \in C(\bar{\Omega})$.

iii) The Perron-Bremermann function $S \psi$ is continuous on $\bar{\Omega}$ for every $\psi \in C(\partial \Omega)$.

We also show that smooth bounded Reinhardt domains in $\mathbb{C}^{2}$ are $c$-regular. However, not all smooth bounded pseudoconvex domains in $\mathbb{C}^{2}$ are $c$-regular. For example, a smooth bounded convex domain whose boundary contains a single analytic disk is not $c$ regular. See also Remark 4 at the end of the paper for a discussion about Hartogs domains in $\mathbb{C}^{2}$.

Theorem 5. Smooth bounded pseudoconvex Reinhardt domains in $\mathbb{C}^{2}$ are c-regular.

Hence on smooth bounded pseudoconvex Reinhardt domains in $\mathbb{C}^{2}$ the plurisubharmonic envelopes of functions continuous up to the boundary are continuous up to the boundary. 
Corollary 2. Let $\Omega$ be a smooth bounded pseudoconvex Reinhardt domain in $\mathbb{C}^{2}$. Then the envelope $E \varphi$ is continuous on $\bar{\Omega}$ for all functions $\varphi \in C(\bar{\Omega})$.

A domain $\Omega$ is B-regular if for any function $f \in C(\partial \Omega)$ there exists a function $u \in$ $\operatorname{PSH}(\Omega) \cap C(\bar{\Omega})$ so that its restriction $\left.u\right|_{\partial \Omega}$ to $\partial \Omega$ is $f$. Sibony [14, 15] showed that a hyperconvex domain is $B$-regular if and only if every boundary point is strongly regular. The bidisk is an example of a $c$-regular domain that is not $B$-regular as the boundary contains analytic disks. It turns out that, for smooth bounded pseudoconvex $c$-regular domains, this is the only obstruction for B-regularity. The precise statement is as follows.

Proposition 1. Let $\Omega$ be a smooth bounded pseudoconvex domain in $\mathbb{C}^{n}$. Then the following statements are equivalent:

i) $\Omega$ is B-regular,

ii) $\partial \Omega$ is strongly regular,

iii) $\partial \Omega$ is c-regular and $\partial \Omega$ has no analytic disks.

iv) The set of strongly regular points is a closed subset of $\partial \Omega$ and $\partial \Omega$ has no analytic disks.

\section{EXAMPLES}

For simplicity we construct examples in $\mathbb{C}^{3}$. However, by a simple modification one can obtain examples in $\mathbb{C}^{n}$ for $n \geq 3$.

Our first example shows that Theorem 4 is not true in $\mathbb{C}^{3}$. We will construct a smooth bounded convex domain $\Omega_{1} \subset \mathbb{C}^{3}$ that is not $c$-regular, yet the set of strongly regular points of $\Omega_{1}$ is closed in $\partial \Omega_{1}$. But first we need a lemma.

Given a bounded domain $\Omega \subset \mathbb{C}^{n}$ and a point $z \in \bar{\Omega}$, we denote by $\mathcal{J}_{z}^{c}=\mathcal{J}_{z}^{c}(\Omega)$ the family of all positive Borel measures $\mu \in C^{*}(\bar{\Omega})$ such that $u(z) \leq \int u d \mu$ for every $u$ in the set $P S H^{c}(\bar{\Omega})$ of all continuous functions on $\bar{\Omega}$ which are plurisubharmonic on $\Omega$. In view of [7, Lemma 2.4], $\mathcal{J}_{z} \subset \widehat{\mathcal{J}}_{z} \subset \mathcal{J}_{z}^{c}$.

Lemma 1. Let $\Omega$ be a smooth bounded domain in $\mathbb{C}^{n}, z \in \bar{\Omega}$ be a point and $f: \overline{\mathrm{D}} \rightarrow \bar{\Omega}$ be a mapping which is holomorphic in an open neighborhood of the closure of the unit disk $\overline{\mathbb{D}}$ so that $f(0)=z$. Then $\mu_{f} \in \mathcal{J}_{z}^{c}$.

Proof. Let $u \in P S H^{c}(\bar{\Omega})$. By [6, Theorem 1] (Sibony [14, Théorème 2.2] proved this result earlier in case of pseudoconvex domains) there are smooth plurisubharmonic functions $u_{j}$ on neighborhoods of $\bar{\Omega}$ that approximate $u$ uniformly on $\bar{\Omega}$. Clearly $u_{j}(z) \leq \int u_{j} d \mu_{f}$ for every $j$. Letting $j \rightarrow \infty$ we get $u(z) \leq \int u d \mu_{f}$. Thus, $\mu_{f} \in \mathcal{J}_{z}^{c}$.

Example 1. We are going to construct a bounded domain $\Omega_{1} \subset \mathbb{C}^{3}$ with smooth boundary such that 
a. the domain $\Omega_{1}$ is convex,

b. the set of strongly regular points of $\Omega_{1}$ is closed,

c. there exists a point in $\partial \Omega_{1}$ which is not $c$-regular.

Let $\psi$ be a non-negative smooth convex function on $[0, \infty)$ such that

i. $\psi=0$ on $[0,1]$

ii. $\psi^{\prime}>0$ and $\psi^{\prime \prime}>0$ on $(1, \infty)$ and $\psi^{\prime}(t)>1$ for $t \geq \sqrt{2}$

iii. $\psi(2)=3$.

Furthermore, let us define

$$
r\left(z_{1}, z_{2}, z_{3}\right)=2 \operatorname{Re}\left(z_{3}\right)+\psi\left(\left|z_{1}\right|^{2}\right)+\psi\left(\left|z_{2}\right|^{2}\right)+\psi\left(\left|z_{3}\right|^{2}\right)+\left|z_{3}\right|^{2}\left(12+a+\left|z_{2}\right|^{2}\right) .
$$

By Sard's Theorem we can choose $a>0$ sufficiently small that the domain

$$
\Omega=\left\{\left(z_{1}, z_{2}, z_{3}\right) \in \mathbb{C}^{3}: r\left(z_{1}, z_{2}, z_{3}\right)<0\right\}
$$

is smooth and bounded. Analysis of the eigenvalues of the Hessian of the function $\left(x_{3}^{2}+\right.$ $\left.y_{3}^{2}\right)\left(12+a+x_{2}^{2}+y_{2}^{2}\right)$ shows that $\left|z_{3}\right|^{2}\left(12+a+\left|z_{2}\right|^{2}\right)$ is convex for $\left|z_{2}\right|<2$. Then $\Omega$ is a smooth bounded convex domain in $\mathbb{C}^{3}$ and the set

$$
\Gamma=\left\{\left(z_{1}, z_{2}, z_{3}\right) \in \mathbb{C}^{3}:\left|z_{1}\right| \leq \frac{1}{2},\left|z_{2}\right| \leq \frac{1}{2}, z_{3}=0\right\} \subset \partial \Omega .
$$

Let $\mathcal{L}_{r}(z ; X)$ denote the Levi form of $r$ at $z \in b \Omega$ applied to the complex tangential vector $X$. It is easy to see that there exist disks in the boundary in $z_{1}$-direction through any point $\left(z_{1}, z_{2}, z_{3}\right) \in \partial \Omega$ where $\left|z_{1}\right|<1$. This means that $\mathcal{L}_{r}\left(z ; e_{1}\right)=0$ for $e_{1}=(1,0,0)$ and $z=\left(z_{1}, z_{2}, z_{3}\right) \in \partial \Omega$ such that $\left|z_{1}\right|<1$ and $z_{3} \neq 0$. Let

$$
W=\left(0,1+\bar{z}_{3}\left(12+a+\left|z_{2}\right|^{2}\right),-\bar{z}_{2}\left|z_{3}\right|^{2}\right) \text {. }
$$

Then $W$ is a complex tangential direction at $z \in \partial \Omega$ near $\Gamma$ that is perpendicular to $e_{1}$. One can calculate that

$$
\begin{aligned}
\mathcal{L}_{r}(z, W)= & \left|z_{3}\right|^{2}\left(\left|1+z_{3}\left(12+a+\left|z_{2}\right|^{2}\right)\right|^{2}-2 \operatorname{Re}\left(z_{2}^{2} \bar{z}_{3}\left(1+\bar{z}_{3}\left(12+a+\left|z_{2}\right|^{2}\right)\right)\right)\right) \\
& +\left|z_{3}\right|^{2}\left(\left|z_{2}\right|^{2}\left|z_{3}\right|^{2}\left(12+a+\left|z_{2}\right|^{2}\right)\right) \\
= & \left|z_{3}\right|^{2}\left(1+O\left(\left|z_{3}\right|\right)\right) \text { for } z \text { near } \Gamma .
\end{aligned}
$$

Then there exists a neighborhood $U$ of $\Gamma$ such that $\mathcal{L}_{r}(z, W)>0$ for $z \in U \backslash \Gamma_{1}$ where $\Gamma_{1}=\left\{\left(z_{1}, z_{2}, z_{3}\right) \in \mathbb{C}^{3}: z_{3}=0\right\}$. Therefore, we showed that there are only analytic disks, one dimensional complex manifolds, in $\partial \Omega$ through $\left(z_{1}, z_{2}, z_{3}\right) \in(U \cap \partial \Omega) \backslash \Gamma_{1}$ such that $\left|z_{1}\right|<1$. 
One can check that $r$ is strongly plurisubharmonic at $\left(z_{1}, z_{2}, z_{3}\right)$ if $\left|z_{1}\right|>1$ and $z_{3} \neq 0$. The fact that there is a disk (given by $\xi \rightarrow\left(e^{i \theta}, \xi, 0\right) \subset \Gamma_{1}$ for $|\xi|<1$ and $\theta \in \mathbb{R}$ ) in $\partial \Omega$ in $z_{2}$-direction through $\left(e^{i \theta}, 0,0\right)$ implies that set of strongly regular points in the boundary of $\Omega$ is not closed (one can approximate $(1,0,0)$ by a sequence $\left\{\eta_{j}\right\} \subset \partial \Omega$ where the norm of the first component of $\eta_{j}$ is strictly larger than 1 and the last component is nonzero). So we need to modify the domain further to make sure that strongly regular points in the boundary are closed. Using Sard's Theorem, again if necessary, we can choose a small $\delta>0$ and a smooth, convex, and non-decreasing function $\lambda: \mathbb{R} \rightarrow[0, \infty)$ such that

i. $\lambda(t)=0$ for $|t| \leq \delta$ and $\min \left\{\lambda^{\prime}(t), \lambda^{\prime \prime}(t)\right\}>0$ for $t>\delta$,

ii. $\min \left\{\lambda\left(\left|z_{1}\right|^{2}+\left|z_{2}\right|^{2}+\left|z_{3}\right|^{2}\right):\left(z_{1}, z_{2}, z_{3}\right) \in \mathbb{C}^{3} \backslash U\right\}>-\min \{r(z): z \in \Omega\}$.

iii. $\Omega_{1}=\left\{\left(z_{1}, z_{2}, z_{3}\right) \in \mathbb{C}^{3}: r\left(z_{1}, z_{2}, z_{3}\right)+\lambda\left(\left|z_{1}\right|^{2}+\left|z_{2}\right|^{2}+\left|z_{3}\right|^{2}\right)<0\right\}$ is a smooth bounded convex domain in $\mathbb{C}^{3}$.

We note that $\Omega_{1} \subset U \cap \Omega$ and the set of weakly pseudoconvex points of $\Omega_{1}$ is

$$
\partial \Omega_{1} \cap\left\{\left(z_{1}, z_{2}, z_{3}\right) \in \mathbb{C}^{3}:\left|z_{1}\right|^{2}+\left|z_{2}\right|^{2}+\left|z_{3}\right|^{2} \leq \delta\right\} .
$$

A point in the boundary of a bounded convex domain in $\mathbb{C}^{n}$ is strongly regular if and only if there are no analytic disks in the boundary through the point (see [5, Proposition 3.2]). Since there is no analytic disk in $\left\{\left(z_{1}, z_{2}, z_{3}\right) \in \mathbb{C}^{3}:\left|z_{1}\right|^{2}+\left|z_{2}\right|^{2}+\left|z_{3}\right|^{2}=\delta\right\}$ we conclude that the set of strongly regular points of $\Omega_{1}$ is

$$
\partial \Omega_{1} \backslash\left\{\left(z_{1}, z_{2}, z_{3}\right) \in \mathbb{C}^{3}:\left|z_{1}\right|^{2}+\left|z_{2}\right|^{2}+\left|z_{3}\right|^{2}<\delta\right\} .
$$

Hence it is closed. However, $\Omega_{1}$ is not $c$-regular. To see this, first notice that there exists a disk in the boundary of $\Omega_{1}$ with center $p=(0,0,0)$ in the $z_{2}$-direction. For example, we can take the analytic disk defined by the map $f(\zeta)=(0, \delta \zeta, 0)$. By Lemma 1 the measure $\mu_{f} \in \mathcal{J}_{z}^{c}$. Let $p_{j}=\left(0,0, w_{j}\right)$ where

$$
w_{j}=-\frac{1}{j}+i \sqrt{\frac{2 j-12-a}{j^{2}(12+a)}} .
$$

One can check that $\left\{p_{j}\right\} \subset \partial \Omega_{1}$ is a sequence converging to $p$ and, by our construction, any analytic disk through $p_{j}$ is in the $z_{1}$-direction.

Claim: If $\mu_{j} \in \mathcal{J}_{p_{j}}$ then $\mu_{j}$ is supported on the disk through $p_{j}$ in $z_{1}$-direction.

Proof of Claim: This can be seen as follows: $\Omega_{1}$ is convex and the transversal direction to each disk through $p_{j}$ is strongly pseudoconvex. Then there exist linear functionals $L_{j}$ such that $L_{j}<0$ on $\bar{\Omega}_{1} \backslash\left\{\left(z_{1}, 0, w_{j}\right) \in \mathbb{C}^{3}: z_{1} \in \mathbb{C}\right\}$ and $L_{j}=0$ on $\left\{\left(z_{1}, 0, w_{j}\right) \in \mathbb{C}^{3}: z_{1} \in \mathbb{C}\right\}$. 
Hence every Jensen measure of $p_{j}$ is supported on the disk through $p_{j}$. This finishes the proof of the claim.

Let $\varphi \in C\left(\bar{\Omega}_{1}\right)$ be a function so that $\varphi$ depends on $z_{2}$ only, $-1 \leq \varphi \leq 0, \varphi(p)=0$, and $\varphi\left(z_{1}, z_{2}, z_{3}\right)=-1$ when $\left|z_{2}\right|=\delta$. Hence $\mu_{f}(\varphi)=-1$. In view of the observation above $\lim _{j \rightarrow \infty} \mu_{j}(\varphi)=\lim _{j \rightarrow \infty} \varphi\left(p_{j}\right)=0$. Thus $\mu_{j}$ does not converge to $\mu_{f}$. From [7, Corollary 4.4] it follows that $p$ is not $c$-regular.

Next we would like to give an example that shows that Theorem 5 is not true in $\mathbb{C}^{3}$. First we need the following proposition whose proof is essentially contained in [10] in the proof of Proposition 6.17. We will therefore skip the proof.

Proposition 2. Let $\Omega$ be a smooth bounded convex domain in $\mathbb{R}^{n}$. Then $\Omega$ has a defining function that is strictly convex on $\Omega$.

Remark 1. Observation of the proof in [10] also shows that a similar statement is true for pseudoconvex domains with plurisubharmonic defining functions. That is, if a smooth bounded pseudoconvex domain $\Omega$ in $\mathbb{C}^{n}$ has a plurisubharmonic defining function then it also has a defining function that is strictly plurisubharmonic on $\Omega$.

Example 2. In this example we will construct a smooth bounded complete Reinhardt pseudoconvex domain in $\mathbb{C}^{3}$ that is not $c$-regular. Hence the statement of Theorem 5 is not true in $\mathbb{C}^{3}$.

Let $H_{1}=\left\{(x, y) \in \mathbb{R}^{2}:-1<x, y<1\right\}$ and $H_{2}$ be a smooth bounded convex domain that is obtained by smoothing out the corners of $H_{1}$ so that $\left\{(x, y) \in \mathbb{R}^{2}: x=1,0 \leq y \leq\right.$ $1 / 2\} \subset \partial H_{2}$. Since $H_{2}$ is convex it has a convex defining function. Then by Proposition 2 we can choose a defining function $\rho$ for $\mathrm{H}_{2}$ that is strictly convex on $\mathrm{H}_{2}$. Let us define

$$
\Omega=\left\{\left(z_{1}, z_{2}, z_{3}\right) \in \mathbb{C}^{3}:\left|z_{3}\right|^{2}+\rho\left(\left|z_{1}\right|^{2},\left|z_{2}\right|^{2}\right)<0\right\} .
$$

Then $\Omega$ is a smooth bounded complete Reinhardt domain in $\mathbb{C}^{3}$. One can show that $\Omega$ is pseudoconvex and has a disk

$$
\Delta=\{(1, \zeta / 2,0): \zeta \in \mathbb{C},|\zeta|<1\}
$$

in the boundary centered at $p=(1,0,0)$ yet all the points on the boundary which belong to the set $R=\left\{\left(z_{1}, z_{2}, z_{3}\right) \in \mathbb{C}^{3}: z_{3} \neq 0\right\}$ are strongly pseudoconvex. Then the set of strongly regular points contains the set $\partial \Omega \cap R$ (strongly pseudoconvex points are strongly regular) yet $p$ is not strongly regular. Hence the set of strongly regular points in $\partial \Omega$ is not closed in $\partial \Omega$. Therefore, Theorem 3 implies that $\Omega$ is not $c$-regular. 


\section{PROOFS}

Proof of Proposition 1, i) and ii) are equivalent by Sibony's results [14, 15]. It is clear that ii) implies iii) and iii) implies iv) by Theorem 3 , So we only need to prove that iv) implies ii). Assume that the set of strongly regular points is closed, $\partial \Omega$ has no analytic disks, and there exists $p \in \partial \Omega$ that is not strongly regular. Then the Levi form of $\Omega$ degenerates at $p$. Since the set of strongly regular points is closed, and not the whole of $\partial \Omega$, the Levi form degenerates on an open set $U$ (in the relative topology) in the boundary of $\Omega$. Then there exists a relatively open set $V \subset U$ in the boundary where the Levi form is of constant rank. This implies that $V$ is locally foliated by complex manifolds (see, for example, [4]). We reach a contradiction. Therefore, $\partial \Omega$ is strongly regular.

The following observation will be needed in the proof of Theorem 4 .

Lemma 2. Let $\Delta$ be the unit disk in $\mathbb{C}, \Omega \subset \mathbb{C}^{2}$ be a bounded domain, and $W$ be a regular bounded domain in $\mathbb{C}$ such that $0 \in \partial W, \bar{\Omega} \subset \Delta \times W$, and $p=(0,0) \in \partial \Omega$. If $\mu \in \widehat{\mathcal{J}}_{p}$, then Supp $\mu \subset \Delta \times\{0\}$.

Proof. Since $W$ is regular there exists a subharmonic function $u$ such that $u(0)=0$ and $u<0$ on $W \backslash\{0\}$. Let $U\left(z_{1}, z_{2}\right)=u\left(z_{2}\right)$. Then $U$ is a plurisubharmonic function on $\Delta \times W$ such that $U(z, 0)=0$ for $z \in \Delta$ and $U<0$ on $\Omega \backslash(\Delta \times\{0\})$. This implies that $\operatorname{Supp} \mu \subset \Delta \times\{0\}$.

We recall from [9] that a bounded domain $\Omega \subset \mathbb{C}^{n}$ is locally $c$-regular if for every point $z \in \partial \Omega$ there is an open neighborhood $N$ of $z$ so that $V=\Omega \cap N$ is $c$-regular. The localization result in [9] says that a bounded domain $\Omega$ is $c$-regular if and only if it is locally c-regular.

Proof of Theorem 4. One direction follows from Theorem 3, For the other direction assume that strongly regular points are closed. If all points in the boundary are strongly regular, then the boundary is $B$-regular. This clearly implies that $\Omega$ is $c$-regular.

Suppose that the set of strongly regular points does not cover the whole boundary. Let $p$ be a boundary point that is not strongly regular. By the localization result in [9] it is enough to prove that there exists an open ball $U$ containing $p$ such that $p$ is $c$-regular in $\Omega_{1}=\Omega \cap U$. Since $\Omega \subset \mathbb{C}^{2}$ and the set of strongly regular points is closed, the Levi form is of constant rank in a neighborhood of $p$ in the boundary. Then we can choose $U$ so that $U \cap \partial \Omega$ is foliated by complex disks.

Using a holomorphic change of coordinates we may assume that $p$ is the origin, the disk $\Delta$ containing $p$ sits in the first coordinate, and positive $y_{2}$-axis is the outward normal 
to the boundary on $\Delta$. Then there exist $r, \theta>0$ so that $\Delta \times\{0\} \subset \bar{\Omega}_{1} \subset \mathbb{C} \times \bar{W}$ where $W=\{z \in \mathbb{C}:|z|<r,|\operatorname{Arg}(z)+\pi / 2|<\theta\}$. Since $W$ is regular Lemma 2 applies and all Jensen measures of $p$ on $\Omega_{1}$ are supported on $\Delta \times\{0\}$. Let $\mu \in \widehat{\mathcal{J}}_{p}$ be a measure in $\Omega_{1}$ and $\left\{q_{j}\right\}$ be a sequence in $\Omega_{1}$ that converges to $p$. Since an open neighborhood (in $\partial \Omega_{1}$ ) of $p$ is foliated by analytic disks the foliation can be parametrized. That is, there exists a smooth mapping $F: \Delta \times(-1,1) \rightarrow \partial \Omega_{1}$ such that $F$ is a diffeomorphism onto its image, $F(z, 0)=z$ on $\Delta$, and $F$ is holomorphic in $z$ (see, for example, Theorem 1.1 and Theorem 2.14 in [4]). Let $\eta$ be the outward normal vector $(0, i)$ to the boundary of $\Omega_{1}$ on the disk $\Delta$. Then there exists a number $a>0$ so that the function $G(z, s, t)=F(z, s)+t \eta$ maps $H=\Delta \times(-a, a) \times(-a, 0]$ into $\bar{\Omega}_{1}$. For any $\alpha \in \Delta$ let

$$
G_{\alpha, \beta}(z, s, t)=G\left(\frac{\beta z+\alpha}{1+\bar{\alpha} \beta z}, s, t\right) \text { for }(z, s, t) \in H .
$$

There exist points $\left(\alpha_{j}, s_{j}, t_{j}\right) \in H$ such that $t_{j}<0$ and $G\left(\alpha_{j}, s_{j}, t_{j}\right)=q_{j}$ for all $j$, and $s_{j} \rightarrow 0$ and $t_{j} \rightarrow 0$ as $j \rightarrow \infty$. We consider $\mu$ as a measure on $\Delta \times\{0\} \times\{0\} \subset H$. Let us choose numbers $0<\beta_{j}<1$ such that $\beta_{j} \rightarrow 1$ and the functions

$$
g_{j}(z)=G_{\alpha_{j}, \beta_{j}}\left(z, s_{j}, t_{j}\right), \quad z \in \bar{\Delta}
$$

map $H$ into $\Omega_{1}$. Let $\mu_{j}=\left(g_{j}\right)_{*} \mu$ (here $\left(g_{j}\right)_{*}$ denotes the push forward by $g_{j}$ ). Since the sequence of functions $\left\{G\left(z, s_{j}, t_{j}\right)\right\}$ converges to $G(z, 0,0)$ uniformly on $\bar{\Delta}$, the sequence of measures $\left\{\mu_{j}\right\}$ converges weak- $*$ to $\mu$ as $j \rightarrow \infty$. Notice that the measures $\mu_{j}$ are supported in $\Omega_{1}$. Moreover for every $j$ we have

$$
\int \varphi d \mu_{j}=\int \varphi \circ g_{j} d \mu
$$

for every $\varphi \in C\left(\bar{\Omega}_{1}\right)$. If $u \in P S H^{b}(\Omega)$, then

$$
u \circ g_{j}(0)=u\left(q_{j}\right) \leq \int u \circ g_{j} d \mu=\int u^{*} d \mu_{j}
$$

for every $j \geq 1$. Hence the measure $\mu_{j} \in \mathcal{J}_{q_{j}}$. This completes the proof of Theorem 4 .

Proof of Theorem [5 Let us assume that $\Omega \subset \mathbb{C}^{2}$ is a smooth bounded pseudoconvex Reinhardt domain, $\theta_{z w}^{1}(t)=\left(e^{i t} z, w\right)$, and $\theta_{z w}^{2}(t)=\left(z, e^{i t} w\right)$. Then $\frac{d}{d t} \theta_{z w}^{1}(t)=\left(i e^{i t} z, 0\right)$ and $\frac{d}{d t} \theta_{z w}^{2}(t)=\left(0, i e^{i t} w\right)$. Assume that $p \in \partial \Omega$ and there exists an analytic disk $\Delta$ in the boundary of $\Omega$ through the point $p$.

First suppose that $p$ is away from coordinate axes. Replacing $\Delta$ by a smaller disk if necessary we may assume that $\Delta$ is away from the coordinate axes as well. Then $\frac{d}{d t} \theta_{z w}^{1}$ and $\frac{d}{d t} \theta_{z w}^{2}$ span $\mathbb{C}^{2}$ on $\Delta$. The fact that $\Delta$ is two real dimensional and that the rotations around $z$ 
and $w$ axes add at least one more dimension imply that there is an open set in the boundary around $p$ that is foliated by analytic disks. We recall that pseudoconvex Reinhardt domains are convexifiable away from the coordinate axes and strongly regular points of a convex domain are precisely the ones with no disks through them in the boundary. Then the set of points in $\partial \Omega$ (away from the coordinate axes) that are not strongly regular is a relatively open set.

Now let us assume that $p$ is a boundary point on the coordinate axes. By the open mapping property of holomorphic functions in one variable there are no nontrivial analytic disks in the intersection of the boundary with the coordinate axes. Hence if there is a disk through $p$ then we can rotate the disk as in the previous case to show that there are disks in a neighborhood of $p$. So without loss of generality assume that $p=(q, 0)$ is in the boundary of $\Omega$ and there is no analytic disk in the boundary of $\Omega$ passing through $(q, 0)$. Then there is a smooth subharmonic function $\phi$ on the disk $\Delta(|q|) \subset \mathbb{C}$ centered at the origin and radius $|q|$ such that $\phi(q)=0$ and $\phi<0$ on $\overline{\Delta(|q|)} \backslash\{q\}$. Let $\psi(z, w)=\phi(z)$. Then $\psi(z, w)<0$ when $|z|<|q|$. However, since there is no analytic disk through $p$ and $\Omega$ is smooth Reinhardt the condition $(z, w) \in \bar{\Omega} \backslash\{p\}$ implies that $|z|<|q|$. Hence, the function $\psi$ peaks at the point $p$.

Therefore, we showed that the set of strongly regular points in $\partial \Omega$ is closed and Theorem 4 implies that $\Omega$ is $c$-regular.

\section{FURTHER REMARKS}

(1) We note that pseudoconvexity is necessary in Proposition 1, Let $B(p, r)$ denote the ball centered at $p$ with radius $r$. Now let $p_{1}=(0,0)$ and $p_{2}=(2,0)$. By smoothing the domain $B\left(p_{1}, 2\right) \backslash B\left(p_{2}, 1\right) \subset \mathbb{C}^{2}$ we can obtain a smooth bounded $c$-regular domain $\Omega$ such that for every $z \in \partial B\left(p_{2}, 1\right) \cap B\left(p_{1}, 2\right) \cap \partial \Omega$ there are disks in $\bar{\Omega}$ passing through $z$. Hence $\Omega$ is not $B$-regular and there are no analytic disks in the boundary.

(2) Whether the cross product of $c$-regular domains is $c$-regular is still an open problem. However, the cross product of two $B$-regular domains is $c$-regular. That is, if $U \subset \mathbb{C}^{n}$ and $V \subset \mathbb{C}^{m}$ are bounded $B$-regular domains then $U \times V$ is $c$-regular. To see this let $p=\left(z_{0}, w_{0}\right) \in \partial(U \times V)$. If $z_{0} \in \partial U$ and $w_{0} \in \partial V$, then there are functions $u \in P S H(U) \cap C(\bar{U}), v \in P S H(V) \cap C(\bar{V})$ so that $u\left(z_{0}\right)=0, v\left(w_{0}\right)=0$, $u(z)<0$ for $z \in \bar{U} \backslash\left\{z_{0}\right\}, v(w)<0$ for $w \in \bar{V} \backslash\left\{w_{0}\right\}$. Then clearly the function $\rho(z, w)=u(z)+v(w)$ is a plurisubharmonic peak function on $U \times V$ for $\left(z_{0}, w_{0}\right)$ and the point $\left(z_{0}, w_{0}\right)$ is strongly regular. So let us assume without loss of generality that $z_{0} \in \partial U$ and $w_{0} \in V$. Let $\mu \in \mathcal{J}_{p}^{c}(U \times V)$. We claim that the support 
of $\mu$ belongs to the set $S=\left\{\left(z_{0}, w\right): w \in V\right\}$. Suppose this is not the case. This means that there is a compact set $K \subset U$ so that $z_{0} \notin K$ and $\mu(K \times \bar{V})>c$ for some $c>0$. Let $u \leq 0$ be a plurisubharmonic peak function on $U$ for $z_{0}$ as above. Let $r(z, w)=u(z)$ for $z \in \bar{U}, w \in \bar{V}$. Then for any $w \in \bar{V}$ we have

$$
0=r\left(z_{0}, w\right)=u\left(z_{0}\right) \leq \int_{K} r d \mu \leq c \max _{K} r<0 .
$$

The contradiction proves the claim that $\operatorname{Supp} \mu \subset S$. Thus $\mu=\delta_{z_{0}} \times v$ for some probability measure $v$ on $\bar{V}$. One can show that $v \in \mathcal{J}_{w_{0}}^{c}(V)$. In fact, if $v \in$ $P S H^{c}(V)$, then let us consider the function $v_{0}(z, w)=v(w)$ defined on the product $U \times V$. One sees that

$$
v\left(w_{0}\right)=v_{0}\left(z_{0}, w_{0}\right) \leq \int v_{0} d \mu=\int v d v .
$$

By [7, Corollary 4.4] we have $\mathcal{J}_{z}^{\mathcal{C}}(V)=\mathcal{J}_{z}(V)$, hence, we see that the measure $\mu \in \mathcal{J}_{p}(U \times V)$. Thus $\mathcal{J}_{p}(U \times V)=\mathcal{J}_{p}^{c}(U \times V)$, and again by [7, Corollary 4.4] the point $p$ is $c$-regular.

(3) Let $U \subset \mathbb{C}^{n}$ and $V \subset \mathbb{C}^{m}$ be domains. Then the set of strongly regular points in $\partial(U \times V)$ is closed if and only if the strongly regular points in $\partial U$ and $\partial V$ are closed. Suppose the strongly regular points in $\partial U$ and $\partial V$ are closed. If $\left(p_{j}, q_{j}\right) \in$ $\partial(U \times V)$ are strongly regular and converging to $(p, q)$, then clearly $p$ and $q$ are strongly regular points in $\partial U$ and $\partial V$ respectively. Let $u(z)$ and $v(w)$ be the plurisubharmonic peak functions in $U$ and $V$ for $p$ and $q$ respectively. The function $s(z, w)=$ $u(z)+v(w)$ is clearly a plurisubharmonic function on $U \times V$ so that $s(p, q)=0$, $s(z, w)<0$ when $(z, w) \neq(p, q)$. Thus, the strongly regular points in $\partial(U \times V)$ are closed.

To prove the other direction assume that the strongly regular points in $\partial(U \times$ $V)$ are closed and without loss of generality assume that $\left\{p_{j}\right\}$ is a sequence of strongly regular points in $\partial U$ that converges to a point $p$. If $q$ is a strongly regular point in the boundary of $V$, then $\left(p_{j}, q\right)$ are strongly regular points in $\partial(U \times V)$ that converges to $(p, q)$. Let $\varphi \leq 0$ be a plurisubharmonic function on $U \times V$ so that $\varphi(p, q)=0, \varphi\left(p^{\prime}, q^{\prime}\right)<0$ when $\left(p^{\prime}, q^{\prime}\right) \neq(p, q)$. Then $u(z)=\varphi(z, q)$ is a plurisubharmonic function on $U$ that peaks at $p$. Hence the set of strongly regular points in $\partial U$ is closed. In a similar way one can show this for $\partial V$.

(4) P. Matheos in his thesis [11] constructed a smooth bounded pseudoconvex complete Hartogs domain in $\mathbb{C}^{2}$ that has no analytic disk in the boundary and yet not $B$-regular. Hence by Proposition 1 this domain is not $c$-regular and there is no analog of Theorem 5 for Hartogs domains. 


\section{ACKNOWLEDGEMENT}

We would like to thank Evgeny Poletsky for valuable comments on a preliminary version of this manuscript.

\section{REFERENCES}

[1] H. J. Bremermann, On a generalized Dirichlet problem for plurisubharmonic functions and pseudo-convex domains. Characterization of Šilov boundaries, Trans. Amer. Math. Soc. 91 (1959), 246-276.

[2] S. Q. Bu and W. Schachermayer, Approximation of Jensen measures by image measures under holomorphic functions and applications, Trans. Amer. Math. Soc. 331 (1992), no. 2, 585-608.

[3] E. Bedford and B. A. Taylor, The Dirichlet problem for a complex Monge-Ampère equation, Invent. Math. 37 (1976), no. 1, 1-44.

[4] M. Freeman, Local complex foliation of real submanifolds, Math. Ann. 209 (1974), 1-30.

[5] S. Fu and E. J. Straube, Compactness of the $\bar{\partial}$-Neumann problem on convex domains, J. Funct. Anal. 159 (1998), no. 2, 629-641.

[6] J. E. Fornæss and J. Wiegerinck, Approximation of plurisubharmonic functions, Ark. Mat. 27 (1989), no. 2, 257-272.

[7] N. G. Göğüş, Continuity of plurisubharmonic envelopes, Ann. Polon. Math. 86 (2005), no. 3, 197-217.

[8] Continuity of plurisubharmonic envelopes, Ph.D. thesis, Syracuse University, 2006.

[9] L L L Lal and global C-regularity, Trans. Amer. Math. Soc. 360 (2008), no. 5, 2693-2707.

[10] A.-K. Herbig and J. D. McNeal, Convex defining functions for convex domains, J. Geom. Anal., 22 (2012), no. 2, 433-454.

[11] P. Matheos, A Hartogs domain with no analytic discs in the boundary for which the $\bar{\partial}-$ Neumann problem is not compact, Ph.D. thesis, University of California Los Angeles, 1997.

[12] E. A. Poletsky, Plurisubharmonic functions as solutions of variational problems, Several complex variables and complex geometry, Part 1 (Santa Cruz, CA, 1989), Proc. Sympos. Pure Math., vol. 52, Amer. Math. Soc., Providence, RI, 1991, pp. 163-171.

[13] _ Holomorphic currents, Indiana Univ. Math. J. 42 (1993), no. 1, 85-144.

[14] N. Sibony, Une classe de domaines pseudoconvexes, Duke Math. J. 55 (1987), no. 2, 299-319.

[15] - Some aspects of weakly pseudoconvex domains, Several complex variables and complex geometry, Part 1 (Santa Cruz, CA, 1989), Proc. Sympos. Pure Math., vol. 52, Amer. Math. Soc., Providence, RI, 1991, pp. 199-231.

[16] J. B. Walsh, Continuity of envelopes of plurisubharmonic functions, J. Math. Mech. 18 (1968/1969), 143148.

(Nihat Gökhan Göğüş) SABANCi University, ORHANLI TUZla, 34956, IstANBul, TuRKey

E-mail address: nggogus@sabanciuniv.edu

(Sönmez Şahutoğlu) University Of TOledo, DePARTMEnt of MATHEMATICs \& STATistics, TOledo, OH 43606, USA

E-mail address: sonmez. sahutoglu@utoledo.edu 